\title{
Effect of pulsation on the near flow field of a submerged water jet
}

\author{
HAREKRISHNA YADAV and AMIT AGRAWAL*
}

Department of Mechanical Engineering, Indian Institute of Technology Bombay, Powai, Mumbai 400076, India

e-mail: krishna.04p@gmail.com; amit.agrawal@iitb.ac.in

MS received 15 June 2017; revised 18 October 2017; accepted 11 December 2017; published online 22 March 2018

\begin{abstract}
The current study investigates the effect of pulsation frequency on the near field characteristics of a submerged water jet using the technique of dye visualization. Flow visualization was performed in water over the range: Reynolds number 540-1540, Strouhal number 0.16-1.75, and at constant amplitude of pulsation of $18 \%$. The results show that the mixing and entrainment process at lower Reynolds number occurs due to diffusion process owing to relatively stable shear layer for the case of a steady jet, whereas the external pulsation promotes an early instability in the shear layer where irregular structures promotes mixing between the jet and surrounding fluids. Images of streaklines show that initial mixing and entrainment processes in the potential core of the jet is due to the development of large vortical structures. While beyond the potential core, mixing and entrainment are governed by the small-scale structures. Further results show that the initiation and growth of vortices in the shear layer depends on the pulse frequency. For a given Reynolds number and amplitude, the number of vortical structures and their size changes with frequency. With an increase in the pulsation frequency, there is an increase in the spreading of the jet along with stretching of the vortical structures. An optimum pulsating frequency at which the effect of pulsation on the flow is maximum occurs at $\mathrm{St}=0.44$, independent of Reynolds number. These results should eventually lead to a better understanding of the physical phenomena responsible for enhanced mixing and entrainment processes in the presence of pulsating jets.
\end{abstract}

Keywords. Submerged water jet; pulsation frequency; dye visualization; entrainment; mixing.

\section{Introduction}

The mixing and entrainment processes are essential features of a jet. The free jet has been studied extensively because of its widespread applications in the field of engineering, such as fuel injection in gas turbines, reciprocating internal combustion engines, industrial type burners, drying of food, cooling processes, and in micro scale applications. Mixing and entrainment characteristics of a steady jet have received considerable attention both theoretically and experimentally [1-4]. These characteristics of the steady jet have been mainly explained through decay in center line velocity, spread rate of half width velocity, and mass flow rate [5]. The entrainment and mixing processes are mainly governed by the initiation of vortex and its downstream movement. Small eddies formed in the shear layer are also responsible for local mixing process [6,7]. The circular jet draws a large amount of fresh fluid from the surrounding medium and produces large scale vortices, owing to the initial instability in the laminar shear layer. These vortices roll-up in the downstream direction of the flow field due to the action of the opposite viscous force. This rolling action enhances the entrainment and mixing process [8-11].

*For correspondence
Crow and Champagne [8] visualized the motion of coherent structures with the help of an optical technique (Schlieren), and also turbulent characteristics of circular jet with/without acoustic excitation. They observed that instability in the jet develops gradually from sinusoid to helix as the jet Reynolds number (Re) is increased from 100 to 1000 . They also determined that the vortex passing frequency at the end of the potential core corresponds to a Strouhal number (St) of 0.3. Popiel and Trass [11] observed the motion of toroidal vortices in the near-field of a free jet with the help of smoke wire visualization. They concluded that during the development phase of vortices, a large amount of fresh fluid is drawn from the surrounding, and the entrainment rate and mixing process are enhanced. Han and Goldstein [12] investigated the flow characteristics of a circular jet with/without acoustic excitation with the help of Schlieren technique. They observed that with acoustic excitation, vortices are formed due to initial instability at larger upstream locations and develop faster than the case without excitations. In addition, they found that the excitation of jet provides a strong and regular vortex pairing process, which in turn gives the vortex passing frequency corresponding to Strouhal number 0.45. Cornaro et al [13] visualized the fluid flow characteristic using a smoke-wire technique and observed low turbulence intensity in the 
shear layer, which promoted organized vortex structure. While high turbulence intensity inhibits the development of organized vortex structure.

In order to observe the effect of flow pulsation on the mixing ability of a jet, Johari and Paduano [14] visualized the flow structure in a gravity driven flow with florescent dye of pulse round jet created by a solenoid valve. They observed, using acid base neutralization reaction, that a pulse jet mixes in a shorter distance as compared to a steady jet. However, this study did not reveal any dependency of pulse jet on its frequency. Otani et al [15] also used a solenoid valve to produce a pulse jet in order to develop an effective dry surface. Their results showed that a pulse air jet was highly effective in drying and cleaning processes. Grosshans et al [16] investigated the mixing characteristics of a pulse jet in a combustion chamber. They concluded that the pulse jet enhances the mixing phenomenon as compared to a steady jet. The injection frequency showed a small effect on mixing in the spray region and no effect of mixing in the atomization region. During the first few injection pulses, considerable enhancement in mixing process was observed with an increase in the injection frequency. Farrington and Claunch [17] investigated the influence of flow pulsation of a planar air jet on its flow structure with the help of infrared imaging and smoke-wire visualization. They observed that the highest mixing occurred at $\mathrm{St}=0.168$ for $\mathrm{Re}=7200$; for $0<\mathrm{St}<0.324$, the structure in the natural jet was smaller and less ordered as compared to a pulsed jet. Further, vortices occurring in the pulsating jet were larger and closer to the nozzle as compared to a steady jet.

In spite of considerable literature being available on submerged jet, the flow characteristics of pulse jet, including the effect of pulsation and amplitude on jet performance have seldom been reported in the literature and hence are not completely understood. In our earlier work [4], PIV measurements were undertaken in the transition and turbulent regimes, whereas in the present work we provide high speed visualization of the flow in the laminar regime. The present work therefore extends our earlier work. The dye visualization technique is employed in this study which has led to a better understanding of the structures in the flow. Flow visualization using dye is an easy, inexpensive and fast method to provide a complete picture of the flow field. The experimental data which is primarily qualitative in nature, was recorded by using a high speed camera. The experiments are conducted over a wide range of Reynolds number and pulsation frequency, and revealed several interesting flow patterns.

\section{Experimental apparatus and data collection}

The free jet study has been performed in a rectangular tank made of $1.3 \mathrm{~cm}$ thick acrylic sheet, with inner dimensions of $70 \times 38 \times 40 \mathrm{~cm}^{3}$. The description of the setup has been provided in our previous studies $[4,18]$. Therefore, here, only some basic features of dye visualization study have been defined. The jet pipe outside and inside diameters are $25 \mathrm{~mm}$ and $20 \mathrm{~mm}$, respectively. A large pipe length of $200 \mathrm{~cm}$ $(\mathrm{L} / \mathrm{D} \approx 100)$ is taken which ensures fully developed flow over the Reynolds number range investigated. The mass flow rate was measured using a magnetic flowmeter (Rosemount, China).

The dye was inserted on one side of the nozzle; this is because the flow is axisymmetric. One hole ( $1 \mathrm{~mm}$ diameter) is drilled on the top surface of the jet nozzle to insert the dye injection needle inside the jet pipe. One bush of inner diameter $0.5 \mathrm{~mm}$ is fixed above the hole using adhesive to regulate the distance of the injected dye from the jet centreline and also to support the needle. The dye injection needle is inserted into the jet pipe through this bush. The needle has an outer diameter of $1 \mathrm{~mm}$ and is sufficiently long so as to ensure a developed flow profile of the dye through them. The dye is injected in the nozzle just before the exit of the jet and below the surface of the nozzle wall. The dye is hung from a height of $150 \mathrm{~cm}$ from the measurement tank by means of a stand and is connected to the injection needles by a rubber pipe. The rubber pipe is installed with valves so as to control the flow of the dye. The injected dye is entrained in the jet flow tracing the streak lines. The dye streak lines were observed and recorded from the front view using a high speed 10 bit camera (Nikon PCO.1200hs). The high speed camera has a maximum frame rate of $1357 \mathrm{fps}$ at $1280 \times 480$ pixels. A white UV lamp source and a $150 \mathrm{~W}$ white halogen lamp are employed for illumination. A white paper was placed on the wall behind and beneath the test section to act as the background and to improve the quality of the recorded images.

A solenoid valve based pulse driver is used to induce pulsations in the flow. Average velocity and nozzle diameter are used in calculation of Reynolds number in each experiment. The Reynolds number is calculated using $\mathrm{Re}=$ $\frac{\mathrm{U}_{\text {avg }} \mathrm{D}}{v}$ where $\mathrm{U}_{\mathrm{avg}}$ is the area average velocity at the nozzle exit and $v$ is the kinematic viscosity of water. In the measurements, pulse amplitude $(\mathrm{A}=18 \%)$ and duty cycle $(\mathrm{DC}=50 \%)$ are kept constant throughout the experiment. The amplitude of pulsation is defined as the ratio of the difference of maximum flow rate $\left(Q_{\max }\right)$ and average flow rate $\left(Q_{a v g}\right)$ to the average flow rate and can written as $\mathrm{A}=\frac{\mathrm{Q}_{\mathrm{max}}-\mathrm{Q}_{\mathrm{avg}}}{\mathrm{Q}_{\text {avg }}}$. The Strouhal number is used to normalize the pulse frequency and defined as $\mathrm{St}=\frac{\mathrm{fD}}{\mathrm{U}_{\text {avg }}}$, where $\mathrm{f}$ represents the pulse frequency.

\section{Results and discussion}

A flow visualization study of several configurations involving submerged axisymmetric jet has been conducted. The flow patterns of the pulsating jet were observed using streaklines, which were obtained by inserting potassium 
permanganate $\left(\mathrm{KMnO}_{4}\right)$ dye. To follow the flow path in the shear layer, dye was injected below the surface of the nozzle wall. Injected dye is entrained in the jet flow thereafter tracing the streaklines. Dye visualization makes the qualitative differentiation between steady jet and pulsating jet. In order to compare the flow structures of steady and pulsating jets, the Reynolds number is kept constant. The experiments were conducted for Reynolds number 540 to 1540 , beyond this Reynolds number, dye diffused chaotically into the flow field making visualization difficult.

The unsteady nature of the pulsating jet is clearly visible from the flow visualization images, where different flow patterns appear even for the same Reynolds number (figure 1). In the case of pulsating jet, flow breaks down and vortex formation is observed (figure $1 \mathrm{~b}, \mathrm{c}, \mathrm{d}$ ), in contrast to flow for a steady jet (figure 1a). The steady jet travels with minimal diffusion over a relatively larger distance as compared to the pulsating jets. In the shear layer of pulsating jet, vortex formation is initiated within the first two diameters of the jet. Pulsation of $0.5 \mathrm{~Hz}(\mathrm{St}=0.44)$ shows vortices having large amplitude which are stretched and diverged in radial direction (figure $1 \mathrm{~b}$ ). Farrington and Claunch [17] reported that the vortices occurring in pulsating jet were larger and closer to the nozzle as compared to steady jet. However for $\mathrm{f}=1 \mathrm{~Hz}$, vortices are formed closer to the nozzle (figure 1c) as compared to $0.5 \mathrm{~Hz}$ case (figure $1 \mathrm{~b}$ ), and are convected in the downstream along the stream-wise direction. Also the stretching and divergence of the vortex formed for $\mathrm{f}=1 \mathrm{~Hz}$ is less than that of vortex formed for $\mathrm{f}=0.5 \mathrm{~Hz}$. One can also observe from these pictures that as the vortex is convected in the downstream direction, it draws the fluid from the surrounding and there is a growth in the vortex size. Crow and Champagne [8] observed that due to the initial instability in the laminar shear layer, a large amount of fresh fluid is drawn from the surrounding medium. This produces a large scale vortex which rolls up downstream and an increase in the mixing and entrainment processes occurs. It is expected that the growth in the size of the vortex would result in an increase in the turbulence intensity, which leads to breaking of the coherent structure into small eddies and generates high level of turbulence. At $2 \mathrm{~Hz}$, vortical structures are not observed (figure 1d); only breaking in dye is observed, which is quite similar to the streaklines observed in a steady jet. The similarity is in terms of formation of vortical structure in steady jet and pulse jet. At other pulse frequency (except $2 \mathrm{~Hz}$ ), streakline showed well-defined vortical structure whereas streakline at steady jet and pulse jet at $2 \mathrm{~Hz}$ does not show a vortical structure.

Figure 1 reveals that the pulsation at $\mathrm{f}=0.5 \mathrm{~Hz}$ $(\mathrm{St}=0.44)$, will lead to a higher spreading rate, entrainment and mixing capability than the other frequencies of pulsation for the same Reynolds number. The enhanced mixing at $\mathrm{f}=0.5 \mathrm{~Hz}$ can be attributed to the presence of ample time for vortex to grow as compared to other frequencies, as the second pulse is generated $2 \mathrm{~s}$ after the first pulse. However for other frequencies, vortices do not get sufficient time to develop; hence they entrain less fluid from the surrounding. Farrington and Claunch [17] showed that the highest mixing occurred at $\mathrm{St}=0.168$ for $\mathrm{Re}=7200$ and $0<\mathrm{St} \ll 0.324$. In order to understand the dependency of optimal pulse frequency on the Reynolds number, experiments are conducted for two additional Reynolds numbers of 1090 and 1540 (figures 2 and 3, respectively). A similar observation is made for these Reynolds numbers as well. Figures 1-3 show that as the Reynolds number changes the location of vortical structure and its size changes even for the same pulse frequency.

Figure 2a shows steady jet structure at $\mathrm{Re}=1090$ compared to the steady jet structure at $\operatorname{Re}=540$; notice that the flow is slightly disturbed at the downstream edge of the view frame for the former case. However, the pulse jet structure at $0.5 \mathrm{~Hz}(\mathrm{St}=0.22)$ is totally changed. It is neither bigger in size nor stretched as compared to the vortex structure at $0.5 \mathrm{~Hz}$ and $\mathrm{Re}=540$. As the pulse frequency is increased to $1 \mathrm{~Hz}(\mathrm{St}=0.44)$, a drastic change in flow structure is observed as shown in figure 2c. The figure shows that the shear layer instability is most dominant for a pulse frequency corresponding to $\mathrm{St}=0.44$. As a result, large spreading of dye and highly stretched vortices are observed. In addition to this, the large structures are destroyed and transformed into small-scale structures. These small-scale structures are mainly responsible for local mixing and entrainment at the surrounding of the jet and fluid interface. In case of pulsation at $\mathrm{f}=2 \mathrm{~Hz}$ $(\mathrm{St}=0.87)$, the vortex formation starts even earlier along the axial direction. Note that vortical structure is not seen at $\mathrm{f}=2 \mathrm{~Hz}(\mathrm{St}=1.75)$ and $\mathrm{Re}=540$ (figure $1 \mathrm{~d}$ ), only small diffusivity of dye is observed. However, the vortices are smaller in size and less stretched as compared to the $1 \mathrm{~Hz}$ pulsation and is somewhat akin to the $0.5 \mathrm{~Hz}$ frequency case. Therefore, interaction of vortical structure to surrounding fluid and core fluid is less when compared to $\mathrm{St}=0.44$. The more interaction of vortical structure leads to decay in the jet center line velocity and shifting of the potential core length towards the upstream. The streak lines captured (not shown) at higher pulse frequency for the same Reynolds number does not show vortical structure, only breaking of dye has been observed.

Figure 3 shows the flow structures at slightly higher Reynolds number $(\operatorname{Re}=1540)$. It is observed that as the pulsation frequency increases from $0.5 \mathrm{~Hz}$ (not shown), the instability in dye increases up to $2 \mathrm{~Hz}$. Also, the initiation of vortical structure is shifted towards the upstream. Further, with the increase in pulsation frequency beyond $2 \mathrm{~Hz}$, it seems that the influence of pulsation in terms of mixing and entrainment decreases. In addition, pulsation corresponding to $\mathrm{f}=2 \mathrm{~Hz}(\mathrm{St}=0.61)$ shows more spreading of dye, and earlier initiation of vortex formation than other pulsation frequencies and steady jet. The formation of vortices at higher frequencies is not only shifted upstream, but also occurrence of quicker formation and earlier 

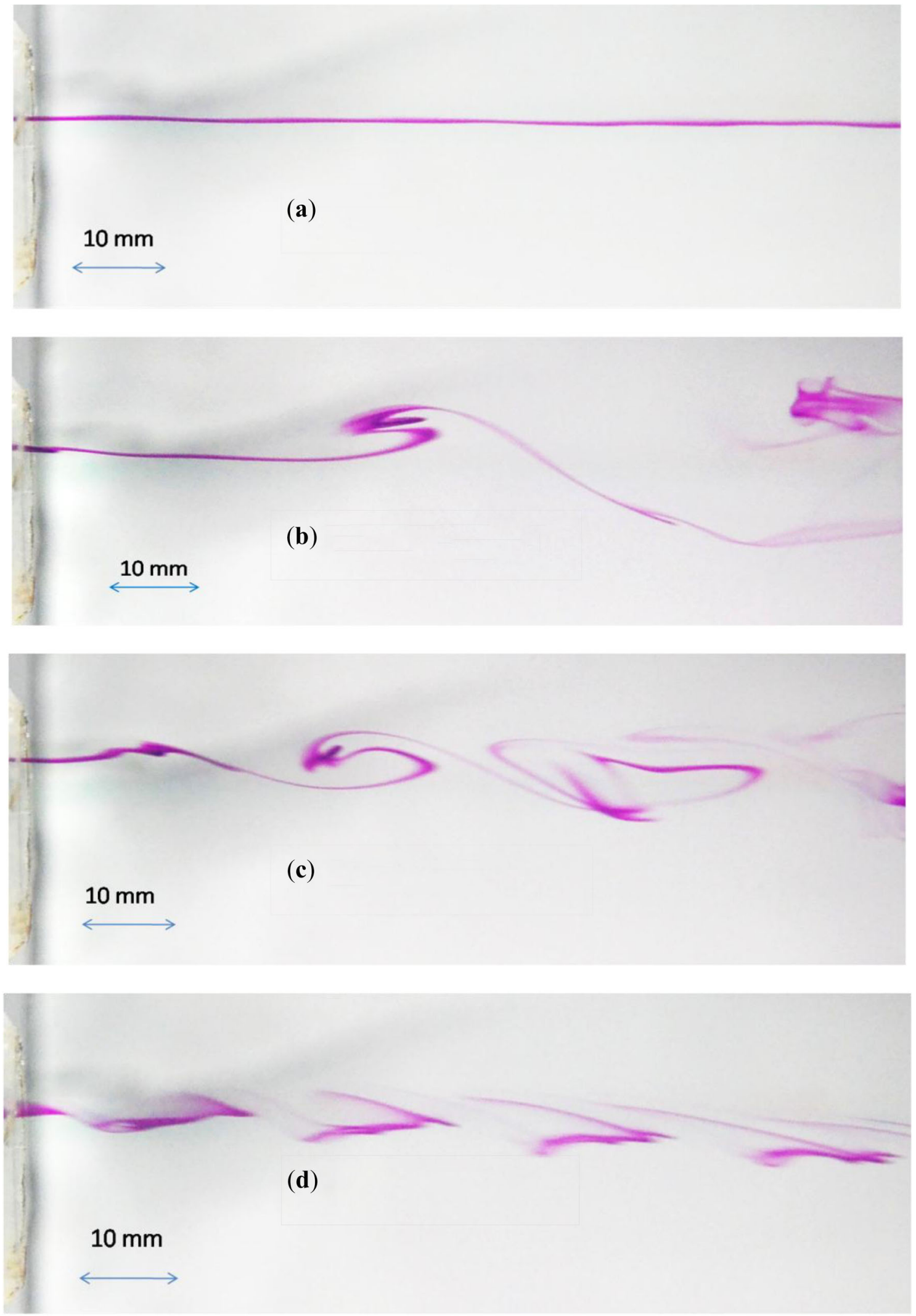

Figure 1. Vortex initiation and development using dye visualization: $\operatorname{Re}=540$. 

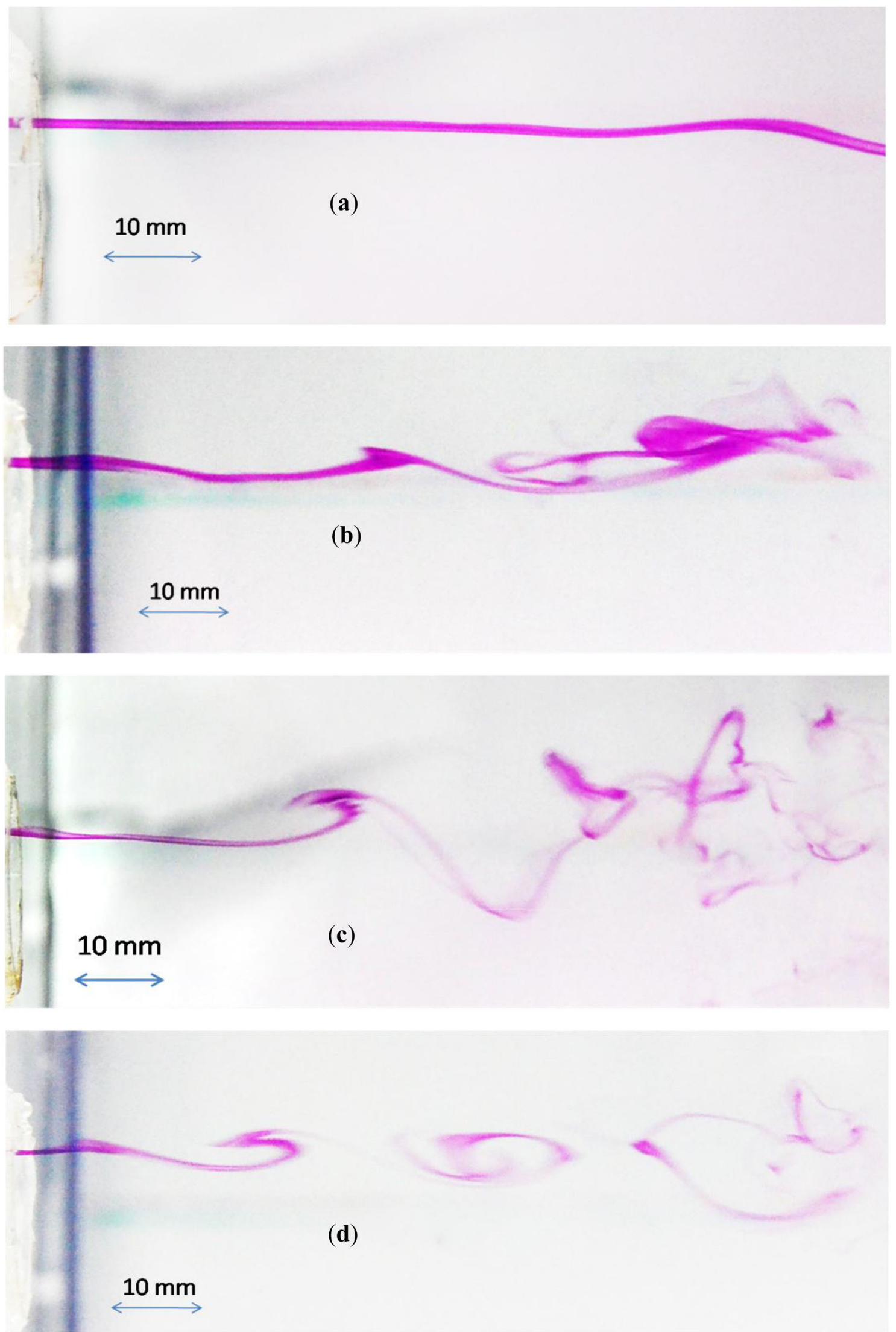

(d)

Figure 2. Vortex initiation and development using dye visualization: $\operatorname{Re}=1090$. 


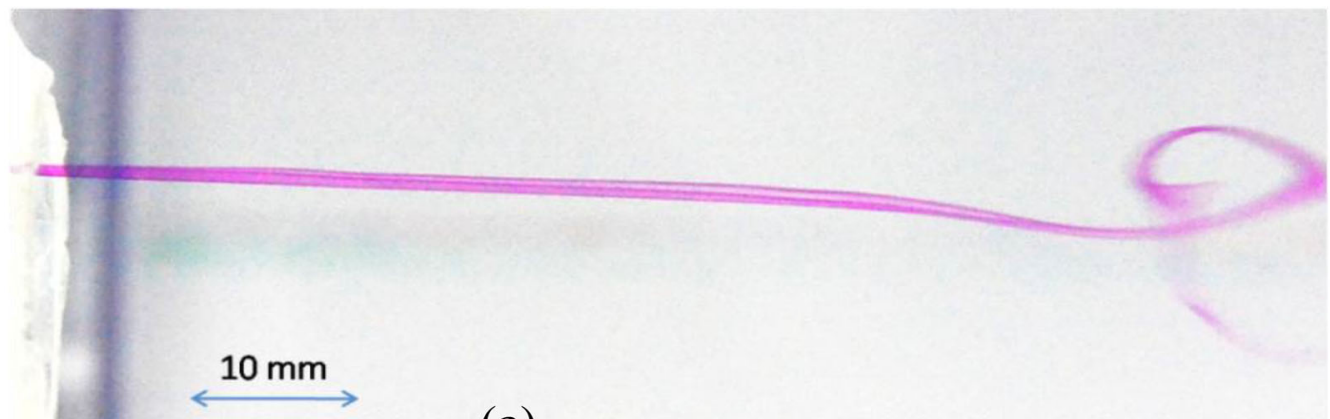

(a)
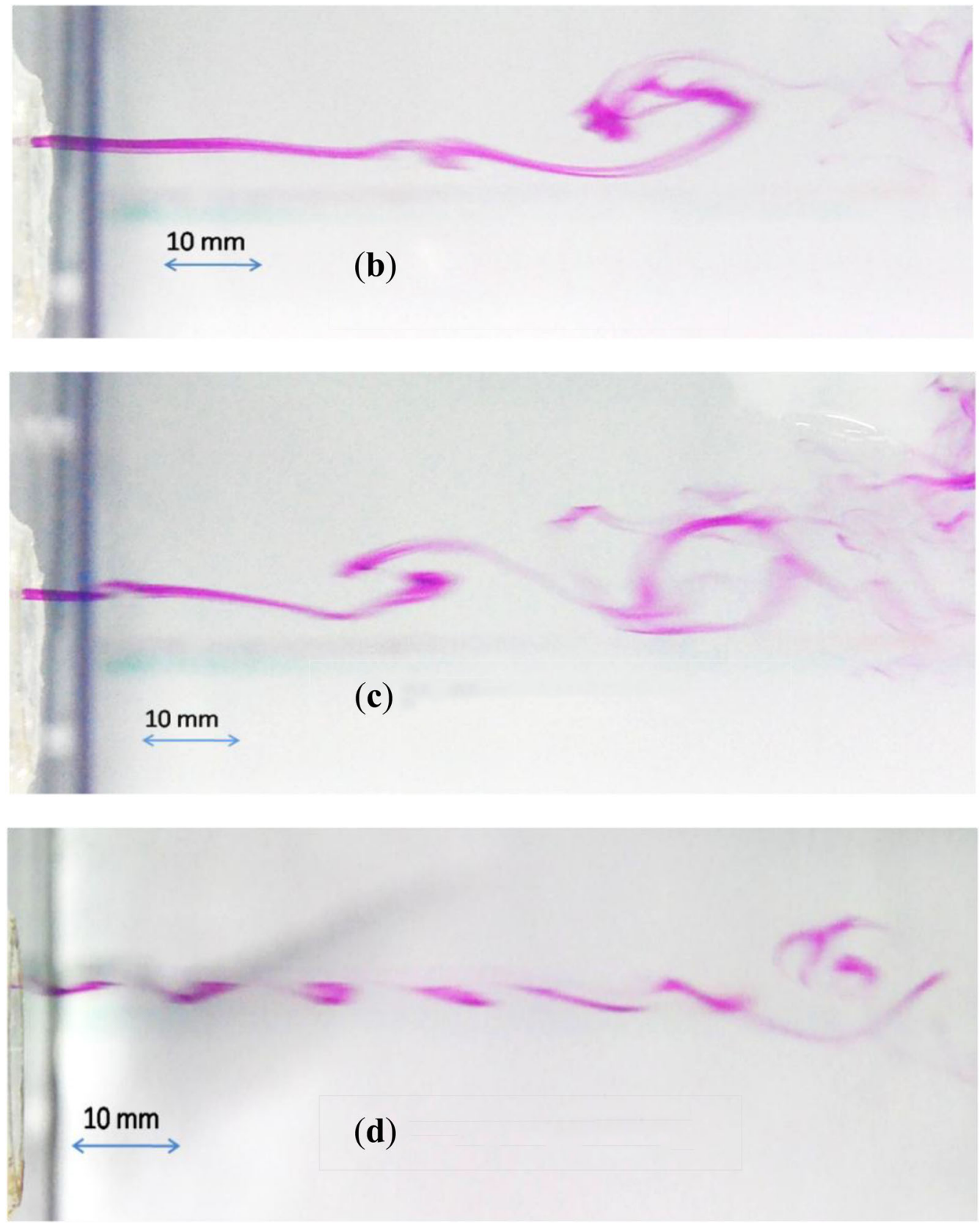

Figure 3. Vortex initiation and development using dye visualization: $\operatorname{Re}=1540$. 
breakdown of vortices take place, which eventually helps in the mixing and entrainment processes. It is predicted that pulsation between 1 and $2 \mathrm{~Hz}$ will provide the maximum pulsation effect at this Reynolds number.

The flow structures depicted at three different Reynolds number (figures 1-3) demonstrate that there exists an optimum frequency, beyond which the instability in the shear layer and vortex strength are not as strong as when they occur at the optimum frequency of pulsation $(\mathrm{St}=0.44)$. For a particular Reynolds number, as the pulsation frequency increases, the number of vortex structure increases in the flow field. Whereas after a certain frequency of pulsation both the size and strength of vortices decrease due to reduced time interval between successive vortex formation [19]. However as the Reynolds number increases the velocity of jet increases, which pulls the surrounding fluid at a faster rate, and the optimum frequency of pulsation shifts towards the higher frequency. In our previous studies $[4,18]$, the results obtained from PIV study shows that for a given Reynolds number and amplitude, the number of vortical structure and their size changes with pulse frequency. With an increase in the pulsation frequency, it has been observed that there is an increase in the spreading of the jet along with stretching of the vortical structures. Further results showed that there exists an optimum pulsating frequency corresponding to $\mathrm{St}=0.44$ at which the effect of pulsation on the flow is maximum. An increase in vortex strength, broadening of the jet, increase in entrainment rate, increase in turbulence intensity, larger decay in jet centerline velocity, shortening of potential core length, are observed at the optimal frequency of pulsation.

It appears from the flow visualization images that both large and small scale vortex structures are present in the flow field. It can be predicted from figures 1-3 that the pulsation corresponding to $0.31<\mathrm{St}<0.88$ will provide better mixing compared to a steady jet. Similar observation was also reported by Yadav et al [4].

The vortex formation frequency of pulse jet is also determined by recording the intensity ratio (intensity of red color to the total intensity) with time at a particular point in the flow. As the vortex passes through the given location, the intensity of red color changes periodically (not shown). The data shows that the intensity of red color indeed changes periodically with time. Similarly, the vortex formation frequency is also observed to be same as the pulse frequency. This result reveals that in each cycle of ON time and OFF time there is formation of a vortical structure. It seems that during the OFF time of a cycle, the jet exits with a higher momentum in a comparatively slow down medium. A viscous force therefore acts on the fluid layer between the jet core fluid and stagnant surrounding fluid. These two opposite forces develop the strain between the fluid layers, which starts to rotate and form the vortical structure. In all the cases it is observed that the frequency of vortex formation is equal to the forcing frequency of the pulse jet.

\section{Conclusions}

The present experimental study has been carried out with the help of dye visualization to understand the behavior of steady and pulsating jets. The photographs obtained from dye visualization enhance the visual inspection and physical insight of the flow characteristics at different pulsation frequencies. The experimental results indicate that for the range of parameters studied, changes in flow structure of the jet are observed.

It is observed that the pulsating jet mixes more rapidly than the steady jet and has a shorter potential core length. There also exists an optimum frequency, beyond which the degree of mixing and vortex strength is not as strong as at the optimum frequency of pulsation. The steady jet shows a relatively smaller spreading. However with pulsating jet, the degree of spreading and stretching in dye gradually increases till an optimal frequency. As the pulsation frequency increases, the number of vortex structure increases in the flow field; whereas after certain frequency of pulsation both the size and strength of vortices decrease due to reduced time interval between successive vortex formations. As the Reynolds number increases velocity of jet increases, which pulls the surrounding fluid at a faster rate, and the optimum frequency of pulsation shifts towards the higher frequency. It is clearly evident from the dye visualization that large amount of mixing and more entrainment from pulsating jets can be obtained in the Strouhal number range of 0.31 and 0.88 , and an optimal frequency may be around Strouhal number of 0.44 .

These results help provide a better picture of pulse jet and change in the structure of vortex with frequency. The identification of an optimal Strouhal number, which appears to be independent of Reynolds number, should help determine the frequency at which pulse jet should be excited to obtain the maximum benefit of pulsation.

\section{References}

[1] Dahm W J A and Dimotakis P E 1987 Measurements of entrainment and mixing in turbulent jets. AIAA Journal 25: 1216-1223

[2] Ricou F P and Spanding D B 1961 Measurement of entrainment by axisymmetric turbulent jet. Journal of Fluid Mechanics 11: 21-23

[3] Rankin G W, Sridhar K, Arulraja M and Kumar K R 1983 An experimental investigation of laminar axisymmetric submerged jets. Journal of Fluid Mechanics 133: 217-231

[4] Yadav H, Agrawal A and Srivastava A 2016 Mixing and entrainment characteristics of a pulse jet. International Journal of Heat and Fluid Flow 61: 749-761

[5] Agrawal A and Prasad A K 2003 Integral solution for the mean flow profiles of turbulent jets, plumes, and wakes. Journal of Fluids Engineering 125: 813-822

[6] Mathew J and Basu A J 2002 Some characteristics of entrainment at a cylindrical turbulence boundary. Physics of Fluids 14: 2065-2072 
[7] Philip J and Marusic I 2012 Large-scale eddies and their role in entrainment in turbulent jets and wakes. Physics of Fluids 24: 055108

[8] Crow S C and Champagne F H 1971 Orderly structure in jet turbulence. Journal of Fluid Mechanics 48: 547-591

[9] Yule A J 1978 Large-scale structure in the mixing layer of a round jet. Journal of Fluid Mechanics 89: 413-432

[10] Zaman K B M Q and Hussain A K M F 1984 Natural largescale structures in the axisymmetric mixing layer. Journal of Fluid Mechanics 138: 325-351

[11] Popiel C O and Trass O 1991 Visualization of a free and impinging round jet. Experimental Thermal and Fluid Science 4: 253-264

[12] Han B and Goldstein R J 2003 Instantaneous energy separation in a free jet. Part I. Flow measurement and visualization. International Journal of Heat and Mass Transfer 46: 3975-3981

[13] Cornaro C, Fleischer A S and Goldstein R J 1999 Flow visualization of a round jet impinging on cylindrical. $E x$ perimental Thermal and Fluid Science 20(2): 66-78
[14] Johari H and Paduano R 1997 Dilution and mixing in an unsteady jet. Experiments in Fluids 23: 272-280

[15] Otani Y, Norikazu N and Hitoshi E 1995 Removal of fine particles from smooth flat surfaces by consecutive pulse air jets. Aerosol Science and Technology 23: 665-673

[16] Grosshans H, Szasz R-Z and Funchs L 2015 Enhanced liquid gas mixing due to pulsating injection. Computers and Fluid 107: 196-204

[17] Farrington R B and Scott D C 1994 Infrared imaging of large-amplitude, low-frequency disturbances on a planar jet. AIAA Journal 32: 317-323

[18] Yadav H, Srivastava A and Agrawal A 2016 Characterization of pulsating submerged jet-A particle image velocimetry study. Journal of Thermal Science and Engineering Applications 8: 011014

[19] Xia Q and Zhong S 2013 Liquid mixing enhanced by pulse width modulation in a Y-shaped jet configuration. Fluid Dynamics Research 45: 025504 